\title{
Cardiology
}

\section{Assessment of Association of rs2200733 SNP on Chromosome 4q25 with the Risk of the Development of Atrial Fibrillation in the Russian Population}

\author{
Vladimir A. Shulman, $\mathrm{PhD}, \mathrm{ScD}^{1}$; Natalya V. Aksyutina, $\mathrm{PhD}, \mathrm{MD}^{1^{*}}$; \\ Elena A. Aldanova, $\mathrm{PhD}, \mathrm{MD}^{1}$; Svetlana Yu. Nikulina, $\mathrm{PhD}, \mathrm{ScD}^{1}$; \\ Vasily V. Kozlov, PhD, MD²; Larissa G. Soinova ${ }^{1}$; Oksana A. Gavrilyuk, $\mathrm{PhD}^{1}$ \\ ${ }^{I}$ Professor V.F. Voino-Yasenetsky Krasnoyarsk State Medical University, Krasnoyarsk, the Russian Federation \\ 2I.M. Sechenov First Moscow State Medical University, Moscow, the Russian Federation
}

\begin{abstract}
The aim of our case-control study was to investigate the possible genetic association of the rs2200733 SNP on chromosome $4 \mathrm{q} 25$ with atrial fibrillation (AF) in the Russian population as this association has not been examined before in this ethnicity.

Methods and Results: A total of 76 unrelated individuals diagnosed with AF and 73 control subjects without any cardiovascular pathology were included in this study. The diagnosis of AF was based on ECG and/or Holter ECG data following standard diagnostic criteria. We found that the TT genotype of the rs 2200733 SNP was associated with a higher risk of AF (OR=1.4, 95\% CI: 1.1-12.4). The homozygote minor rare allele genotype TT of the rs2200733 SNP tended to elevate the risk of lone AF development (OR=2.5, 95\% CI: 1.2-19.5). A risk of secondary AF development did not depend on the rs $2200733 \mathrm{SNP}$ on chromosome $4 \mathrm{q} 25$ (OR=0.5, 95\% CI: 0.2-1.3).

Conclusion: Our results provide additional evidence for the association between the rs2200733 (4q25) SNP on chromosome $4 \mathrm{q} 25$ and AF, emphasizing the need for further studies examining the role of this polymorphism in AF. (International Journal of Biomedicine. 2018;8(4):280-283.)
\end{abstract}

Key Words: atrial fibrillation • single nucleotide polymorphism • chromosome 4q25 • rs2200733・odds ratio

\section{Abbreviations}

AF, atrial fibrillation; GWASs, genome-wide association studies; SNP, single nucleotide polymorphism; Afl, auricular flutter; OR, odds ratio; CI, confidence interval.

\section{Introduction}

Recently, a lot of emphasis has been given to personalized medicine. In this connection, there are being conducted various types of research aimed at studying the genetic predictors of different disorders in the heart rhythm and cardiac conduction system. ${ }^{(1,2)}$ The advent of GWASs

*Corresponding author: Natalya V. Aksyutina, PhD, MD. Krasnoyarsk State Medical University n.a. Prof. V.F. Voino-Yasenetsky, Krasnoyarsk, the Russian Federation. E-mail: aks-n-v@yandex.ru has provided great insight into the molecular mechanisms implicated in $\mathrm{AF}^{\left({ }^{(3-7)}\right.}$ Detecting new genetic predictors of $\mathrm{AF}$ is of great importance as this heart rhythm disorder is one of the most widespread (1-2\% of the population) and dangerous due to its complications. ${ }^{(8,9)}$ Thus, every fifth stroke is an AFrelated stroke, and the mortality among AF patients is twice as high independent on other risk factors. ${ }^{(8,9)}$

In the majority of cases, AF occurs on the background of various cardio-vascular diseases and syndromes, more often on the background of hypertension, ischemic heart disease, mitral heart disease and others. However, in one-third of cases, 
people without any pathology develop AF. In such cases, this is known as lone AF.

GWASs for AF have led to the identification of novel variants that appear to confer increased susceptibility to sporadic AF. Among these, the common variant rs 2200733 on chromosome $4 \mathrm{q} 25$ has been strongly and independently associated with an increased risk of $\mathrm{AF}$ in various ethnicities. (7,10-18) $^{(18)}$

The identified gene nearest rs2200733 SNP is PITX2, which encodes the transcription factor Pitx2c (paired-like homeodomain transcription factor 2, isoform c). While the functional implications of $4 \mathrm{q} 25$ variants are poorly understood, the proximity of the locus to PITX2 presents an intriguing potential pathophysiological link to $\mathrm{AF} .{ }^{(19)}$ Reduced expression of Pitx2c, a key regulator of left-right asymmetry, has recently been linked to atrial fibrillation. ${ }^{(20)}$

Heterozygous deletion of Pitx $2 \mathrm{c}$, the cardiac isoform of Pitx2, in mice is sufficient to provoke increased inducibility of atrial fibrillation without obvious structural cardiac alterations, ${ }^{(21,22)}$ associated with a shortening of the left atrial action potential duration. ${ }^{(21)}$ There is a marked chamber specificity of Pitx2c expression in the adult heart: mRNA transcripts are expressed almost 100-fold higher in the left as compared to the right adult human and murine atrium. ${ }^{(21)}$

P. Kahr et al. revealed systematic differences between left and right atrial gene expression and supports the hypothesis that Pitx2c has a functional role in maintaining "leftness" in the atrium in adult murine and human hearts. ${ }^{(20)}$ M.J. Kolek et al. found that a common 4q25 AF susceptibility allele (rs2200733) is associated with PR interval prolongation in patients with lone and typical AF and controls with no AF. Given that prolonged PR interval is an established risk factor for $\mathrm{AF}$, this observation, in the context of previously described functional effects of PITX2 deficiency, provides further knowledge about the pathophysiological link of 4q25 variants with $A F .{ }^{(20)}$

L. Shi et al. ${ }^{(18)}$ carried out case-control association studies with $383 \mathrm{AF}$ patients versus 851 non-AF controls and 811 ischemic stroke patients versus 688 non-stroke controls to assess the association between rs 2200733 and AF as well as that between rs2200733 and ischemic stroke in a mainland Chinese Han population. Highly significant association was detected between rs2200733 and AF in a Chinese Han population (allelic $P=3.7 \times 10\left({ }^{-11}\right)$ with $\mathrm{OR}=1.81$; genotypic $P=4.1 \times 10\left({ }^{-12}\right)$ with a dominant model). Moreover, significantly stronger association was found with lone $\mathrm{AF}\left(\mathrm{OR}=2.40, P=1.3 \times 10\left(^{-9}\right)\right.$ compared to $\mathrm{OR}=1.59, P=6.2 \times 10\left({ }^{-7}\right)$ for other types of AF; $P=0.02$ for two ORs).

K.T. Lee et al. found that the Taiwanese with the CC genotype of the rs2200733 SNP remained recessively associated with a lower risk of developing AF than those with the TT genotype (OR=0.27, 95\% CI: $0.11-0.65 ; P<0.01){ }^{(16)}$

To test the polymorphisms on chromosome $4 \mathrm{q} 25$, $16 \mathrm{q} 22$ and $1 \mathrm{q} 21$ in a group of patients (pts) that underwent catheter ablation of $\mathrm{AF}, 410$ patients with $\mathrm{AF}$ that underwent pulmonary vein isolation were included in the study performed by M. Kiliszek et al. ${ }^{(7)}$ Control group $(\mathrm{n}=550)$ was taken from healthy population, matched for age, sex and presence of hypertension. The study showed that the T allele of rs2200733 favored the increased number of episodes of AF per month $(P=0.045)$ and larger pulmonary vein diameter (recessive model, $P=0.032$ ) in Polish population.

Opposite results were obtained in Danish research performed by K.M. Henninqsen et al. ${ }^{(23)}$ In this study, authors investigated the association of rs2200733 and lone AF in 196 young patients. Results suggested that rs 220733 was not a risk factor for $\mathrm{AF}$ in patients with no other cardiovascular disease and with early onset of the arrhythmia.

M.S. Olesen et al. ${ }^{(6)}$ investigated 8 SNPs in 209 patients with early-onset lone AF and 534 individuals free of AF. They found that three SNPs, rs2200733 (4q25), rs3807989 (7p31), and rs $11047543(12 \mathrm{p} 12)$, were associated with early-onset lone $\mathrm{AF}$ (OR=1.62, 95\% CI: 1.16-2.27; $P=0.004$ for rs2200733).

A. Ferran et al. ${ }^{(11)}$ analyzed the association between two genetic variants (rs2200733 and rs7193343) in a Spanish population and the risk of developing atrial fibrillation. A case-control study included 257 case patients with AF and 379 controls. rs2200733 SNP was associated with a higher risk of $\mathrm{AF}(\mathrm{OR}=1.87,95 \% \mathrm{CI}: 1.30-2.70)$.

J.D. Roberts et al. ${ }^{(24)}$ sought to characterize the association between rs2200733 and prevalent Afl (atrial flutter) and to determine if the variant could predict $\mathrm{AF}$ after cavotricuspid isthmus ablation. Authors performed a genetic association study of 295 patients with Afl and/or AF and 469 controls using multivariable logistic regression. The rs2200733 rare allele was associated with an adjusted 2.06-fold increased odds of isolated Afl (95\% CI: 1.13-3.76; $P=0.019)$ and an adjusted 2.79-fold increased odds of a combined phenotype of $\mathrm{AF}$ and Afl (95\% CI: 1.81-4.28; $P<0.001)$.

In the study performed by K. Kalinderi et al. ${ }^{(17)}$, the T/T genotype and the T allele of the rs2200733 SNP were detected more frequently in patients with AF compared to controls ( $13.2 \%$ vs. $2.3 \%, P=0.001$, and $29.6 \%$ vs. $17.9 \%, P=0.001$ ), suggesting that the rs 2200733 SNP increases susceptibility to $\mathrm{AF}$ in the Greek population.

In the study performed by A. Bhanushali et al., ${ }^{(25)}$ the rs $2200733 \mathrm{~T}$ allele was associated with the risk of lone $\mathrm{AF}(\mathrm{OR}=2.80,95 \% \mathrm{CI}: 1.08-7.24 ; P=0.042)$. F. Chen et al. ${ }^{(26)}$ demonstrated that rs 2200733 was strongly associated with $\mathrm{AF}$ recurrence after ablation $(P=0.011)$ and the minor allele $\mathrm{T}$ increased the risk for recurrence $(\mathrm{OR}=1.715)$. The patients with genotype TT had larger size of right atrium and superior pulmonary veins than those of CC genotype.

Data regarding a connection between the rs 2200733 SNP and AF in different populations are contradictory: Some researchers show a statistically significant association with $\mathrm{AF}$, in particular lone $\mathrm{AF}$, whereas other studies have not found this association.

The aim of our case-control study was to investigate the possible genetic association of the rs2200733 SNP on chromosome 4q25 with AF in the Russian population as this association has not been examined before in this ethnicity.

\section{Materials and Methods}

A total of 76 unrelated individuals (41 men and 35 women) diagnosed with AF and 73 control subjects (38 men 
and 35 women) without any cardiovascular pathology were included in this study. The diagnosis of AF was based on ECG and/or Holter ECG data following standard diagnostic criteria. A paroxysmal form of AF was diagnosed in $82.9 \%$ of patients, and a permanent form of AF was diagnosed in $17.1 \%$ of patients.

All patients were divided into two groups: Group 1 comprised 33 (43.4\%) patients diagnosed with lone AF, Group 2 - $43(56.6 \%)$ patients whose principal disease was hypertension (37.2\%), coronary heart disease (Class II-III angina pectoris $(44.2 \%))$ or both diseases (18.6\%).

The median age in the groups did not differ significantly (52 years $[44.0 ; 63.0]$ and 52 years $[45.5 ; 63.5]$, respectively).

All patients underwent the following examinations: ECG, echocardiography, Holter ECG, exercise stress test, transesophageal stimulation of the left atrium, and blood test for thyroid hormones. All participants were genotyped for the presence of the rs2200733 SNP using real-time polymerase chain reaction.

The present study was approved by the local Ethics Committee of Professor V.F. Voino-Yasenetsky Krasnoyarsk State Medical University. Written informed consent was obtained from each patient.

Statistical analysis was performed using SPSS v. 20.0 (SPSS Inc, Chicago, IL). For descriptive analysis, results are presented as median (Me) and interquartile range (IQR; 25th to 75th percentiles). Deviation from HardyWeinberg equilibrium and differences in allele distributions between the two groups were assessed by $\chi 2$ - test with 1 degree of freedom (df), whereas differences in genotype distributions between cases and controls were assessed by the $\chi 2$ - test with $2 \mathrm{df}$. The odds ratio (OR) and their $95 \%$ confidence intervals (CI) were calculated to estimate the strength of the association. For all tests, a probability value of $P<0.05$ was considered statistically significant.

\section{Results and Discussion}

We found that the TT genotype of the rs2200733 SNP was associated with a higher risk of $\mathrm{AF}(\mathrm{OR}=1.4,95 \% \mathrm{CI}$ : 1.1-12.4) (Fig.1).

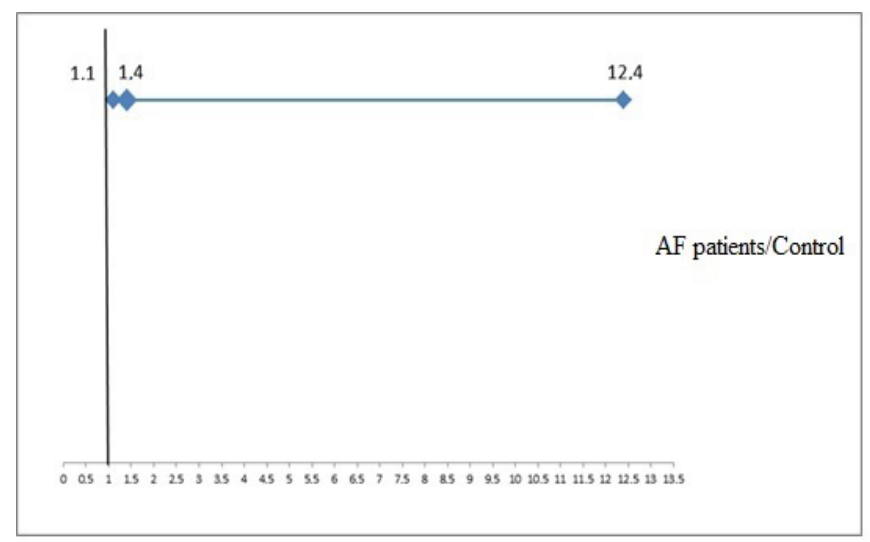

Fig. 1. OR for the rs2200733 SNP genotype frequencies in AF patients (CC $+C$ T relative to $T T)$.
The homozygote minor rare allele genotype TT of the rs2200733 SNP tended to elevate the risk of lone AF development ( $\mathrm{OR}=2.5,95 \%$ CI: 1.2-19.5) (Fig. 2). In Figures 1 and 2, we can see that $95 \% \mathrm{CI}$ does not include value 1 (y-axis), which gives evidence that the revealed link is statistically significant.

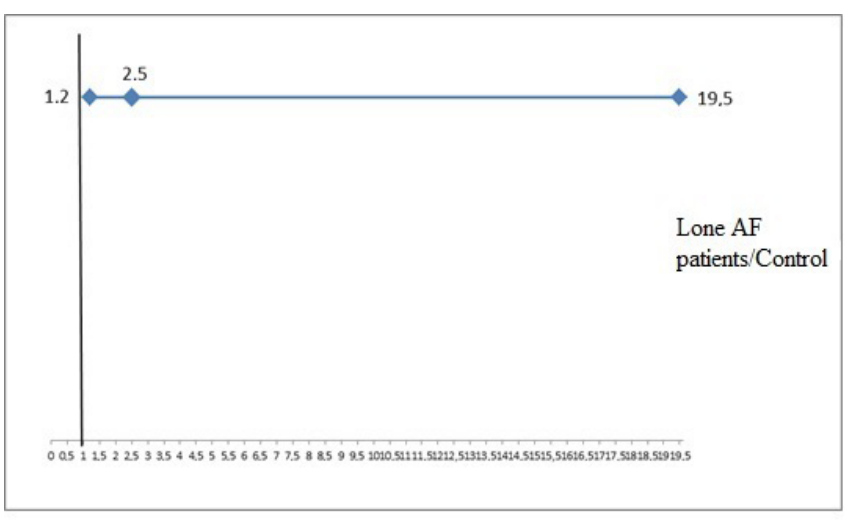

Fig. 2. OR for the rs2200733 SNP genotype frequencies $(C C+C T$ relative to $T T)$ in patients with lone $A F$.

A risk of secondary AF development did not depend on the rs $2200733 \mathrm{SNP}$ on chromosome $4 \mathrm{q} 25$ (OR $=0.5,95 \% \mathrm{CI}$ : $0.2-1.3$ ) ( Fig. 3).

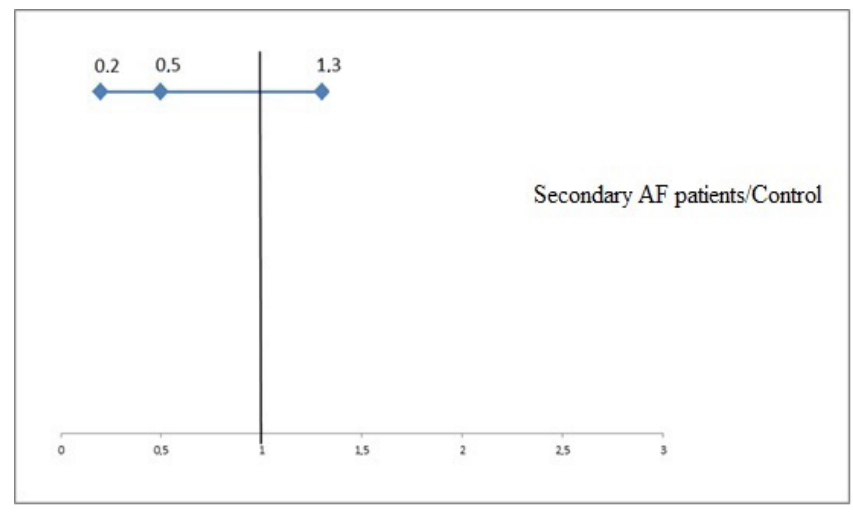

Fig. 3. OR for the rs2200733 SNP genotype frequencies $(C C+C T$ relative to $T T)$ in patients with secondary $A F$.

The present study showed that a TT genotype of the rs 2200733 SNP on chromosome 4q25 was strongly associated with the risk of lone $\mathrm{AF}(\mathrm{OR}=2.5,95 \% \mathrm{CI}$ : 1.2-19.5). The results of our research, obtained on the Russian population for the first time, are similar to those obtained earlier by a number of authors. Thus, L. Shi et al. found a stronger association of TT genotype of the rs2200733 SNP with lone AF (OR=2.40, $\left.P=1.3 \times 10^{-9}\right) .{ }^{(18)} \mathrm{M}$. Olesen et al. found that the rs $2200733 \mathrm{SNP}$ was associated with early-onset lone $\mathrm{AF}(\mathrm{OR}=1.62,95 \% \mathrm{CI}$ : 1.16-2.27; $P=0.004){ }^{(6)} \mathrm{J}$. Roberts et al. revealed that the rare $\mathrm{T}$ allele was associated with an adjusted 2.06-fold increased odds of isolated Afl $(95 \% \mathrm{CI}$ : 1.13-3.76, $P=0.019)$ and an adjusted 2.79-fold increased odds of a combined phenotype of AF and Afl (95\% CI: 1.81-4.28, $P<0.001){ }^{(24)} \mathrm{F}$. Chen et al. demonstrated that rs2200733 was strongly associated with 
$\mathrm{AF}$ recurrence after ablation $(P=0.011)$ and the minor allele $\mathrm{T}$ increased the risk for recurrence $(\mathrm{OR}=1.715){ }^{(26)}$

Thus, the results obtained are in line with reports of previous studies carried out in different European and Asian populations. Our results provide additional evidence for the association between the rs2200733 (4q25) SNP on chromosome 4q25 and AF, emphasizing the need for further studies examining the role of this polymorphism in AF.

\section{Conflict of interest}

The authors declare that they have no competing interests.

\section{References}

1. Nikulina SY, Schulman VA, Kuznetsova OO, Aksjutina NV, Shesternja PA, Chernova AA, et al. [Clinical and genetic pecularities of atrial fibrillation]. Rational Pharmacotherapy in Cardiology. 2008;4(2):13-8. [Article in Russian].

2. Nikulina SY, Schulman VA, Kuznetsova OO, Aksjutina NV, Chernova AA, et al. [Atrial fibrillation: the genealogy and genetics]. Siberian Medical Review. 2008;52(4):4-10. [Article in Russian].

3. Tsai CT, Lai LP, Hwang JJ, Lin JL, Chiang FT. Molecular genetics of atrial fibrillation. J Am Coll Cardiol. 2008;52(4):241-50. doi: 10.1016/j.jacc.2008.02.072.

4. Judge DP. The complex genetics of atrial fibrillation. J Am Coll Cardiol. 2012;60(13):1182-4. doi: 10.1016/j. jacc.2012.04.031.

5. Ellinor PT, Yi BA, MacRae CA. Genetics of atrial fibrillation. Med Clin North Am. 2008;92(1):41-51, x.

6. Olesen MS, HolstAG, Jabbari J, Nielsen JB, Christophersen IE, Sajadieh A, et al. Genetic loci on chromosomes 4q25, 7p31, and $12 \mathrm{p} 12$ are associated with onset of lone atrial fibrillation before the age of 40 years. Can J Cardiol.2012;28(2):191-5. doi: 10.1016/j.cjca.2011.11.016.

7. Kiliszek M, Franaszczyk M, Kozluk E, Lodzinski P, Piatkowska A, Broda G, et al. Association between variants on chromosome 4q25,16q22 and 1q21 and atrial fibrillation in the Polish population. PLoS One. 2011;6(7):e21790. doi: 10.1371/journal.pone.0021790.

8. Diagnosis and treatment of atrial fibrillation. Recommendation of RCS, ASSC and ACS. Russian J Cardiol. 2013;4s(3).

9. Lloyd-Jones ДМ, Wang ТJ, Leip EP, Larson MG, Levy D, Vasan RS, et al. Lifetime risk for development of atrial fibrillation: the Framingham Heart Study. Circulation. 2004;110(9):1042-6.

10. Gudbjartsson DF, Arnar DO, Helgadottir A, Gretarsdottir $\mathrm{S}$, Holm H, Sigurdsson A, et al. Variants conferring risk of atrial fibrillation on chromosome 4q25. Nature. 2007;448(7151):353-7.

11. Ferran A, Alegret JM, Subirana I, Aragonès G, LluisGanella C, Romero-Menor C, et al. Association between rs2200733 and rs7193343 genetic variants and atrial fibrillation in a Spanish population, and meta-analysis of previous studies. Rev Esp Cardiol (Engl Ed). 2014;67(10):822-9. doi: 10.1016/j.rec.2013.12.019.

12. Mohanty S, Santangeli P, Bai R, Di Biase L, Mohanty P, Pump A, Natale A. Variant rs2200733 on chromosome 4q25 confers increased risk of atrial fibrillation: evidence from a metaanalysis. J Cardiovasc Electrophysiol.2013;24(2):155-61. doi: 10.1111/jce. 12017 .
13. Goodloe AH, Herron KJ, Olson TM. Uncovering an intermediate phenotype associated with rs2200733 at 4q25 in lone atrial fibrillation. Am J Cardiol.2011;107(12):1802-5. doi: 10.1016 /j.amjcard.2011.02.326.

14. Lubitz SA, Sinner MF, Lunetta KL, Makino S, Pfeufer A, Rahman R, et al. Independent susceptibility markers for atrial fibrillation on chromosome4q25. Circulation.2010;122(10):97684. doi:10.1161/CIRCULATIONAHA.109.886440.

15. Viviani Anselmi C, Novelli V, Roncarati R, Malovini A, Bellazzi R, Bronzini R, et al. Association of rs2200733 at 4q25 with atrial flutter/fibrillation diseases in an Italian population. Heart. 2008;94(11):1394-6. doi: 10.1136/hrt.2008.148544.

16. Lee KT, Yeh HY, Tung CP, Chu CS, Cheng KH, Tsai WC, et al. Association of RS2200733 but not RS10033464 on $4 q 25$ with atrial fibrillation based on the recessive model in a Taiwanese population. Cardiology. 2010;116(3):151-6. doi: $10.1159 / 000318172$.

17. Kalinderi K, Fragakis N, Koskinas KC, Katritsis D, Letsas K, Efremidis M, et al. Association Between rs2200733 Polymorphism on Chromosome 4q25 and Atrial Fibrillation in a Greek Population. Hellenic J Cardiol. 2015;56(3):224-9. 18. Shi L, Li C, Wang C, Xia Y, Wu G, Wang F, et al. Assessment of association of rs2200733 on chromosome 4q25 with atrial fibrillation and ischemic stroke in a Chinese Han population. Hum Genet. 2009;126(6):843-9. doi: 10.1007/s00439-009-0737-3.

19. Kolek MJ, Parvez B, Muhammad R, Shoemaker MB, Blair MA, Stubblefield T, et al. A common variant on chromosome $4 \mathrm{q} 25$ is associated with prolonged PR interval in subjects with and without atrial fibrillation. Am J Cardiol. 2014;113(2):30913. doi: 10.1016/j.amjcard.2013.08.045.

20. Kahr PC, Piccini I, Fabritz L, Greber B, Schöler H, Scheld $\mathrm{HH}$, et al. Systematic analysis of gene expression differences between left and right atria in different mouse strains and in human atrial tissue. PLoS One. 2011;6(10):e26389. doi: 10.1371/journal.pone.0026389.

21. KirchhofP, Kahr PC, Kaese S, Piccini I, Vokshi I, Scheld HH, et al. PITX2c is expressed in the adult left atrium, and reducing Pitx2c expression promotes atrial fibrillation inducibility and complex changes in gene expression. Circ Cardiovasc Genet. 2011;4(2):123-33. doi: 10.1161/CIRCGENETICS.110.958058. 22. Wang J, Klysik E, Sood S, Johnson RL, Wehrens XH, Martin JF. Pitx2 prevents susceptibility to atrial arrhythmias by inhibiting left-sided pacemaker specification. Proc Natl Acad Sci U S A. 2010 May 25;107(21):9753-8. doi: 10.1073/ pnas. 0912585107.

23. Henningsen KM, Olesen MS, Haunsoe S, Svendsen JH. Association of rs2200733 at 4q25 with early onset of lone atrial fibrillation in young patients. Scand Cardiovasc J. 2011;45(6):324-6. doi: 10.3109/14017431.2011.594081.

24. Roberts JD, Hsu JC, Aouizerat BE, Pullinger CR, Malloy MJ, Kane JP, et al. Impact of a 4q25 genetic variant in atrial flutter and on the risk of atrial fibrillation after cavotricuspid isthmus ablation. J Cardiovasc Electrophysiol. 2014;25(3):271-7. doi: 10.1111/jce.12317.

25. Bhanushali A, Nair A, Jagdale G, Suvarna T, Das BR. Association of Genetic Variants at the 4q25 Locus with Atrial Fibrillation in Indian Population. J Clin Lab Anal. 2017 Jan;31(1). doi: 10.1002/jcla.22017.

26. Chen F, Yang Y, Zhang R, Zhang S, Dong Y, Yin X, et al. Polymorphism rs2200733 at chromosome $4 \mathrm{q} 25$ is associated with atrial fibrillation recurrence after radiofrequency catheter ablation in the Chinese Han population. Am J Transl Res. 2016;8(2):688-97. 